Lecture Notes

Juntendo Medical Journal

2014. $60(6), 576-579$

\title{
Radiation Effects on Human Beings and Radiological Protection
}

\author{
KEISUKE SASAI* \\ * Department of Radiation Oncology, Juntendo University Graduate School of Medicine, Tokyo, Japan
}

\begin{abstract}
In this review, I would like to discuss the effects of ionizing radiation on human beings, and protection against it. $\mathrm{X}$-rays or $\gamma$-rays are electromagnetic radiation with short wavelengths, and have sufficient energy to cause the ejection of one or more orbital electrons from an atom or molecule. This process is called ionization, and this kind of radiation is referred to as ionizing radiation. There are other high-energy particles that have an ionizing effect, and they are all regarded as ionizing radiation. Ionizing radiation can be artificially made, or originate from radioisotopes or space.

Ionizing radiation can cause cell death or genomic mutation, which is related to carcinogenesis. Only ionizing radiation over the threshold dose can cause symptoms related to cell death (deterministic effects), whereas we suppose that there is no threshold for carcinogenesis (stochastic effects) by radiation in terms of safety. A dose limitation has been established for occupational and public exposure, with the aim of preventing deterministic effects and reducing risks of stochastic effects to the extent reasonably achievable. Radiation therapy is one of the most useful treatment modalities for cancers. Currently, we can use many modern treatment modalities in radiation therapy in addition to standard external-beam therapy.
\end{abstract}

Key words: ionizing radiation, radiation therapy, sievert, gray, radiation dose limit

In this review, I would like to discuss the effects of ionizing radiation on human beings, and protection from it.

\section{Ionizing radiation and radioisotopes}

You may be able to identify seven colors in a rainbow. Visible lights are electromagnetics, a kind of wave, and their color depends on the wavelength. If the wavelength is shorter than $400 \mathrm{~nm}$, the light is invisible and is called ultraviolet. Lights of shorter wavelength than ultraviolet are called X-rays or $\gamma$-rays ${ }^{1)}$. They have sufficient energy to ionize an atom or molecule, and they are referred to as ionizing radiation. There are other high-energy particles which have an ionizing effect, and they are

Table-1 Examples of ionizing radiation

\begin{tabular}{lccl}
\hline \multicolumn{1}{c}{ Particle } & Mass ${ }^{*}$ & Charge $^{* *}$ & \multicolumn{1}{c}{ Comments } \\
\hline Photon & 0 & 0 & electromagnetics, X-rays, $\gamma$-rays \\
Proton & 1 & +1 & Proton beams \\
Neutron & 1 & 0 & Neutron beams \\
Electron & 0.00055 & -1 & $\beta$-rays, electron beams \\
${ }^{4} \mathrm{He}$ & 4 & +2 & $\alpha$-rays \\
${ }^{12} \mathrm{C}$ & 12 & +6 & Carbon ion beams \\
\hline
\end{tabular}

*: Atomic masses are given in terms of the mass of one isotope of oxygen (mass number 16), which is arbitrarily assigned the value 16.000 .

$* *: 1.6 \times 10^{-19} \mathrm{C}$

\section{Keisuke Sasai}

Department of Radiation Oncology, Juntendo University Graduate School of Medicine

2-1-1 Hongo, Bunkyo-ku, Tokyo 113-8421, Japan

TEL: +81-3813-3111 E-mail: ksasai@juntendo.ac.jp

〔Received Aug. 29, 2014〕 
all regarded as ionizing radiation. Table-1 lists examples of ionizing radiation. We can create ionizing radiation artificially by accelerating charged particles ${ }^{1)}$. Ionizing radiation also comes from radioisotopes or outside of this planet.

\section{In what way do we receive ionizing radiation?}

Some radioisotopes have natural origins, and others are man-made. Potassium- $40\left({ }^{40} \mathrm{~K}\right)$ has a very long half-life $(1,250,000,000$ years $)$, and irradiates high-energy $\beta$-rays and $\gamma$-rays. It makes up $0.01 \%$ of the total amount of natural potassium. Another example is carbon-14 $\left({ }^{14} \mathrm{C}\right)$, which is produced high in the sky. Carbon-14 has a half-life of 6000 years, and its concentration in the atmosphere has been constant for thousands of years.

There are $4000 \mathrm{~Bq}$ of ${ }^{40} \mathrm{~K}$ and $2500 \mathrm{~Bq}$ of ${ }^{14} \mathrm{C}$ in our body (https://www.remnet.jp/lecture/forum/ 02.pdf), and this means that we are constantly irradiated by ourselves. There are many other radiation sources around us. The average radiation dose received by a human from natural radiation is $2.4 \mathrm{mSv}$ per year (range 1-13 mSv) : inhalation of radon gas 1.26 (0.2-10) $\mathrm{mSv}$, external terrestrial $0.48(0.3-1)$ $\mathrm{mSv}$, ingestion $0.29(0.2-1) \mathrm{mSv}$, and cosmic radiation $0.39(0.3-1) \mathrm{mSv}^{2)}$. However, sizeable population group receives $10-20 \mathrm{mSv}$.

Since the first atomic bomb experiment in 1945, humans have been contaminating the environment with many kinds of artificial radioisotopes, which also irradiate us.

\section{Quantities used in the field of ionizing radiation}

We usually use 3 major dosimetric quantities in the field of ionizing radiation as shown in Figure-1. Radioisotopes disintegrate continually. The rate of disintegration depends on the total number of radioisotope nuclei. Therefore, the quantity of radioisotopes is represented by how many nuclei decay per second. This is called radioactivity, and the unit is a "becquerel (Bq)" ${ }^{3}$.

If radiation comes in contact with our body, we absorb its energy. This is called absorbed dose and is defined as the absorption of one joule of radiation energy per one kilogram of matter, represented as $\mathrm{J} / \mathrm{kg}$, or gray $(\mathrm{Gy})^{3)}$.

There is another unit, sievert (Sv), which is not a

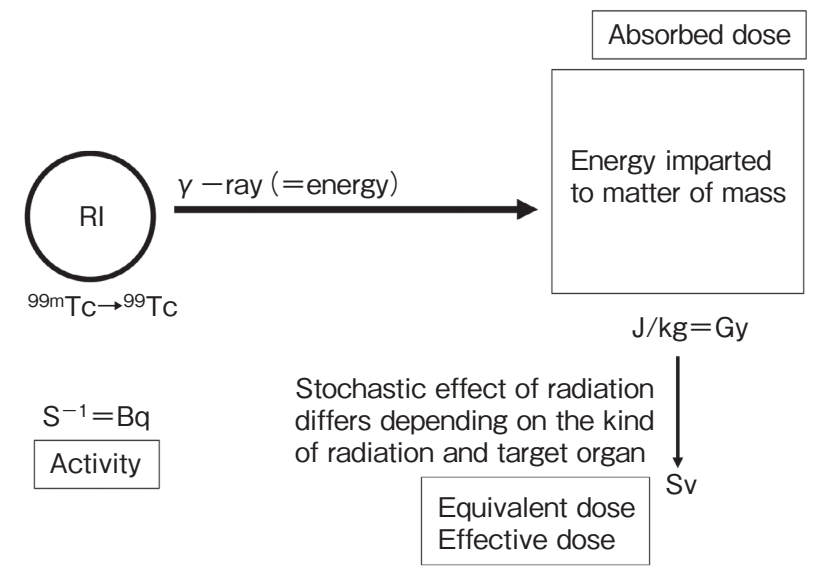

Figure-1 Three major dosimetric quantities in the field of radiation

pure unit of physics, and is used for the purpose of radiation protection. This unit can be used to indicate two measurements: equivalent dose and effective dose. Various kinds of ionizing radiation cause cancers of varying probability. Therefore, the International Commission on Radiological Protection (ICRP) designates a factor between 1 and 20 for each kind of ionizing radiation. The absorbed dose in the unit of Gy is multiplied by this factor (radiation weighting factor) in order to calculate the equivalent dose $^{3)}$.

If we receive radiation in our body homogeneously, it causes cancers in varying probability in each organ. Therefore, the ICRP provides a different number, the tissue weighting factor, which is representative of the probability of cancer and heritable effects (stochastic effects) for each organ ${ }^{3)}$. There is no evidence of hereditary effects of radiation in human beings. The effective dose is the sum of the equivalent dose to the organ multiplied by the tissue weighting factor of the tissue.

\section{Radiation effects on human beings}

Ionizing radiation causes several kinds of DNA damage. Among these, DNA double-strand breaks are most likely related to cell death ${ }^{4)}$ ). Just after the occurrence of DNA double-strand breaks, repair of the damage begins. Almost all of these aberrations can be repaired correctly; however, some are incorrectly repaired, which may result in cell death or carcinogenesis.

More than a particular number of cell deaths in 
Table-2 The characteristics of deterministic and stochastic effects

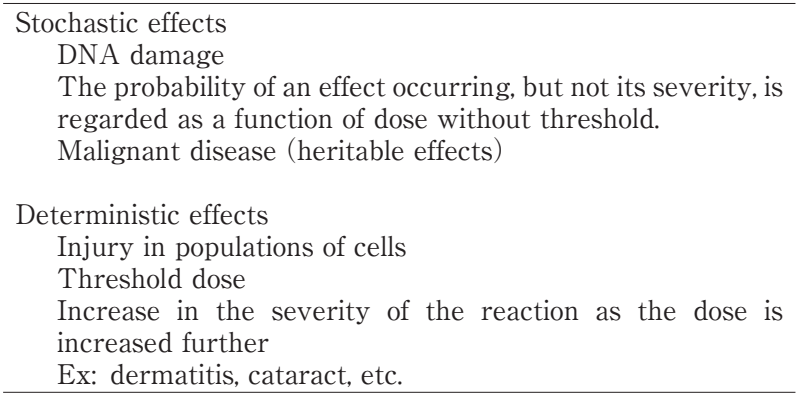

an organ can cause symptoms. In other words, ionizing radiation over the threshold dose can cause symptoms. All phenomena except for carcinogenesis belong to this type, and are called deterministic effects ${ }^{3)}$.

In the case of carcinogenesis, it is supposed that the number of misrepaired radiation-induced DNA double-strand breaks is proportional to the dose without threshold. Although there is no apparent evidence of increased risk of cancer in patients who received less than $100 \mathrm{mSv}$ of radiation, we assume that even a lower dose can cause cancer without threshold for safety purposes. Table- 2 shows the characteristics of deterministic and stochastic effects.

The thresholds for deterministic effects in almost all organs are around $5 \mathrm{~Gy}$. However, those of some organs are low: the ovaries, testes, bone marrow, and lens are much more radiosensitive ${ }^{3)}$.

The effects of ionizing radiation on the intrauterine embryo or fetus are deterministic effects, and the thresholds are over $100 \mathrm{mGy}$. The estimated doses for the fetus from various radiological examinations of the mother are much lower than these thresholds. Therefore, artificial abortion is a contraindication in almost all cases of radiation exposure to the fetus during medical examinations.

\section{Radiation dose limitation}

There are 3 categories of radiation exposure, namely occupational exposure, public exposure, and medical exposure, in addition to exposure from natural environmental ionizing radiation ${ }^{3)}$. During the early days of using ionizing radiation, many researchers and workers died of radiation damage. In 1928, the first dose limitation was established to protect these workers from radiation-induced injuries ${ }^{3)}$. Since then, there have been many efforts to improve the regulations of radiation protection. The latest limits of planned exposure to ionizing radiation are as follows ${ }^{3)}$ : limit of effective dose for occupational exposure is $100 \mathrm{mSv}$ per 5 years with the further provision that the effective dose should not exceed $50 \mathrm{mSv}$ in any single year. Additional restrictions apply to occupational exposure of pregnant women. That for public exposure is $1 \mathrm{mSv}$ in a year. Annual equivalent dose limits in lens of the eye, skin and hands and feet for occupational exposure are $150 \mathrm{mSv}, 500 \mathrm{mSv}$ and $500 \mathrm{mSv}$, respectively. While the limits in lens of the eye, and skin and hands for public exposure are $15 \mathrm{mSv}$ and $50 \mathrm{mSv}$, respectively. The limits do not apply to emergency exposure situations, where an informed, exposed individual is engaged in volunteer life-saving activity or is attempting to prevent a catastrophic situation.

\section{Radiation therapy}

Radiation therapy is one of the most useful treatment modalities for malignant diseases. The therapy can save the organ from which cancer arises, and is available to aged patients or patients of low performance status. Currently, we can use many modern treatment modalities in radiation therapy, in addition to standard external-beam therapy, such as high LET beam therapy, brachytherapy, and intravenous or oral administration of radioisotopes.

As mentioned above, ionizing radiation gives its energy to materials when it travels through them. The rate of energy absorption per traveling length depends on the nature of the ionizing radiation ${ }^{6}$. This is called linear energy transfer, which is represented by the unit of $\mathrm{keV} / \mu \mathrm{m}$. X-rays and $\gamma$-rays, electron beams, and proton beams have low LET values, with a range of $0.1-5 \mathrm{keV} / \mu \mathrm{m}$, whereas those of $\alpha$-rays, neutron beams, and carbon ion beams are higher ${ }^{7)}$. Ionizing radiation with a high LET value has specific characteristics in comparison with that with a low LET value; 1) much direct damage to DNA; 2) high biological effects on cells; 3) low dependence on oxygen concentration; 4) low dependence on cell cycle; and 5) less repair capacity from DNA damage ${ }^{6 / 7)}$. Therefore, it is probable that 
Table-3 Kinds of radiation used in radiation therapy

\begin{tabular}{cll}
\hline \multicolumn{1}{c}{ Radiation } & Physical characteristics & Biological effect \\
\hline Photons & Deep structures & \\
Electron beams & Surface structures & Similar to photons \\
Neutron beams & Similar to photons & Higher than photons \\
Proton beams & Bragg peak * & Similar to photons \\
Carbon ion beams & Bragg peak & Higher than photons \\
\hline *: The dose deposited by a beam of monoenergetic protons or carbon ions increases slowly with \\
depth, but reaches a sharp maximum near the end of the particle's range in the Bragg peak.
\end{tabular}

high LET radiation can achieve good treatment results.

If we can focus the beams on the target volume, we may effectively eradicate these tumors. Proton beams and carbon ion beams provide very precise dose conformity, because the dose deposited by a beam of mono-energetic protons or carbon ions increases slowly with depth, but reaches a sharp maximum near the end of the particle's range in the Bragg peak. Furthermore, carbon ion beams are high LET radiation ${ }^{8)}$. Table-3 shows the characteristics of various kinds of radiation used in radiation therapy.

Carbon ion beam and proton beam facilities are becoming more prevalent; 4 carbon and 8 proton facilities are available here in Japan (http://www. nirs.go.jp/rd/collaboration/himac/laboratory.shtml). However, it costs about 4-15 billion yen to build such a facility. We should test the effectiveness of particle therapy as soon as possible.

\section{Conclusion}

There are many natural radiation sources around us.

Ionizing radiation causes both determinative and stochastic effects.

Radiation therapy is one of the most useful treatment modalities for cancers. Currently, we can use many modern treatment modalities in radiation therapy in addition to standard external-beam therapy.

\section{References}

1) Johns HE, Cunningham JR: The physics of radiology 3rd ed. Springfield: Charles C Thomas, 1980: 1-134.

2) United Nations Scientific Committee on the Effects of Atomic Radiation: Sources and effects of ionizing radiation UNSCEAR 2008 Report to the General Assembly with Scientific Annexes. 2008.

3) Valentin J: The 2007 recommendations of the International Commission on Radiological Protection. Annals of the ICRP ICRP publication 103. 2007.

4) Hall EJ, Giaccia AJ: Molecular mechanisms of DNA and chromosome damage and repair In: Radiobiology for the Radiologist 7th ed. Philadelphia: Lippincotte Williams \& Wilkins, 2012: 12-34.

5) Wouters BG, Begg A: Irradiation-induced damage and the DNA damage response. In: Joiner MC, van der Kogel A, eds. Basic Clinical Radiobiology 4th ed. London: Hodder Arnold, 2009: 11-26.

6) Joiner MC: Linear energy transfer and relative biological effectiveness. In: Joiner MC, van der Kogel A, eds. Basic Clinical Radiobiology 4th ed. London: Hodder Arnold, 2009: 68-77.

7) Hall EJ, Giaccia AJ: Linear energy transfer and relative biological effectiveness In: Radiobiology for the Radiologist 7th ed. Philadelphia: Lippincotte Williams \& Wilkins, 2012: 104-113.

8) Doer W, Joiner MC: Protons and other ions in radiotherapy. In: Joiner MC, van der Kogel A, eds. Basic Clinical Radiobiology 4th ed. London: Hodder Arnold, 2009: 332-338. 